\title{
INSTALLMENT OPTIONS CLOSE TO EXPIRY
}

\author{
G. ALOBAIDI AND R. MALLIER \\ Received 6 December 2005; Revised 5 June 2006; Accepted 31 July 2006
}

We use an asymptotic expansion to study the behavior of installment options close to expiry. Installment options are contracts where the price is paid over the life of the option rather than as a lump sum at the time of purchase, and where the contract can be allowed to lapse at any time. Series solutions are obtained for the location of the free boundary and the price of the option.

Copyright (C) 2006 G. Alobaidi and R. Mallier. This is an open access article distributed under the Creative Commons Attribution License, which permits unrestricted use, distribution, and reproduction in any medium, provided the original work is properly cited.

\section{Introduction}

In the last thirty years, there has been a dramatic growth in the trading of options, which are contracts between two parties giving one party the right but not the obligation to partake in a financial transaction with the other party at or before a specified date in the future. The majority of options involve the right to buy or sell an underlying asset at a prescribed price, known as the strike or exercise price. An option carrying the right to buy an asset is a call, while the right to sell is a put. One way to classify options is according to exercise style. It is straightforward to price European options, which can only be exercised at expiry, using the Black-Scholes option pricing formula [6]. American options, which can be exercised at any time at or prior to expiry, are harder to price because the possibility of early exercise leads to a free boundary separating the region where it is optimal to hold from that where exercise is optimal. In theory, exercise should take place only on this free boundary, known as the optimal exercise boundary. This sort of free boundary problem is common in diffusion problems such as melting and solidification problems and is referred to as a Stefan problem, and a large number of studies have focused on the optimal exercise boundary for American options, and on the behavior of this boundary close to expiry, including the studies in $[1,2,5,13,16,17,20,21,25]$.

In addition to standard American and European options, known as vanilla options, there exist numerous exotic options, which have either unusual payoffs or some other 
unusual features. One exotic is the installment option, for which the purchase price is paid over the life of the option rather than at the time of purchase. To keep the contract alive, the holder must continue to pay the premiums, although he has the option of terminating the contract by halting the payments, in which case the payoff is zero. This ability to abandon the option can be viewed as an American-style early exercise feature, and leads to a free boundary problem similar to that arising for vanilla Americans.

The earliest article on a standard installment option seems to be [15], while more recently $[9,10]$ considered no-arbitrage bounds and robust and static hedging for installment options. Reference [9] focused on discrete installments, but did touch on the continuous case considered here, and also presented numerical simulations, and discussed the importance of installment options. Installment options are also mentioned in $[7,14]$. In [4], we used a partial Laplace transform to derive an integral equation for the location of the free boundary for an installment option, which we solved close to expiry.

Installment options can be found embedded in other contracts, including many life insurance contracts, and are also in common use in the financing of capital projects, with some examples given in [12], where the authors use the phrase sequential investment. One model from the world of real options is that due to [19], in which a firm must invest in a project continuously and receives no payoff until the project is complete. While the model of [19] bears many resemblances to an installment option, it also had some differences, notably that in an installment option, if an investor ceases to pay the premiums, the option lapses, while in [19], the project can be resumed at a later time without loss of the earlier capital outlays.

In our analysis, we will use a simple model of an installment option [35], in which the price $S$ of the underlying asset obeys a log-normal random walk, and the premium on the contract is paid continuously at a rate $L$, so in time $d t$ the owner pays $L d t$, while the project pays a continuous dividend yield to the holder. The payoff at expiry is that of a vanilla European.

The right to abandon means that the value can never be negative, and this leads to a free boundary problem, similar to that for vanilla American options. The holder of an installment option would obviously allow the contract to lapse if the expected net present value of the contract were negative, while he would continue to pay the premiums if the net expected present value were positive, and so it follows that the value of the option must be zero on the free boundary, with the investor holding the installment option on one side of the boundary and the zero portfolio on the other. In addition, we have the usual smooth pasting or high contact condition [24] that the option's delta (or derivative of its value with respect to asset price) must be continuous across the boundary. Since the zero portfolio is held on one side of the boundary, this means that the delta must be zero at the boundary.

In the present study, we will use a technique developed by Tao [26-34] for free boundary problems arising in melting and solidification. Tao used a series expansion in time to find the location of the moving surface of separation between two phases of a material, and in almost all of the cases he studied he found that the location of the interface was proportional to $\tau^{1 / 2}, \tau$ being the time since the two phases were first put in contact. To apply Tao's method, we will first use a change of variables $[11,35]$ to transform 
the governing equations into the heat conduction equation studied by Tao, along with a nonhomogeneous term. This approach has been taken for vanilla American options in the past $[1,2,11,21]$. We will present our analysis for installment options in Section 2, followed by a brief discussion of our results in Section 3.

\section{Analysis}

From [35], the price $V(S, t)$ of an installment option is governed by the partial differential equation $(\mathrm{PDE})$

$$
\frac{\partial V}{\partial t}+\frac{\sigma^{2} S^{2}}{2} \frac{\partial^{2} V}{\partial S^{2}}+(r-D) S \frac{\partial V}{\partial S}-r V-L=0
$$

The term $-L$, which is not present in the usual Black-Scholes-Merton PDE $[6,23]$ for equity options, represents the continual input of cash via the premium: in a time $d t$ an amount $L d t$ must be paid to keep the option alive. $S$ is the price of the underlying and $t$ the time. The risk-free rate $r$, the dividend yield $D$, and the volatility $\sigma$ are assumed constant. This equation is valid for $t<T$, the expiration date of the option, and we will assume that the payoff of an installment option held to maturity is that of a vanilla European option, $\max (S-E, 0)$ for a call and $\max (E-S, 0)$ for a put, where $E$ is the strike price of the option. It is also only valid where it is advantageous to continue to pay the premiums, and must be solved together with the appropriate boundary conditions at the free boundary, which itself is unknown and must be solved for. At the free boundary, a portfolio consisting of an installment option is exchanged for the zero portfolio. In free boundary problems such as the present study as well as vanilla American options, the conditions at the free boundary are that the value of the portfolio and its delta, or derivative with respect to stock price $(\partial V / \partial S)$, must be continuous across the free boundary. The condition on the value of the portfolio is straightforward, and was discussed in Section 1, but that on the delta is less straightforward, and is known as the high contact condition of Samuelson [24]. Although there does not appear to have been a proof of the high contact condition specifically for installment options, the arguments in [24] can be extended to this case, as can the derivation in [18] of the condition for vanilla American options using the first derivative condition for a maximum point amongst possible functional forms for the free boundary. For an installment option, since the option is abandoned at the free boundary, the conditions there become

$$
V=\frac{\partial V}{\partial S}=0
$$

The condition on the delta $(\partial V / \partial S)$ merits further comment. At expiry, where it is optimal to hold the option, $(\partial V / \partial S)=1$ for the call and -1 for the put, yet on the free boundary we have $(\partial V / \partial S)=0$ for $\tau>0$, so that there is a discontinuity in the delta. A similar discontinuity occurs for the vanilla American call with $D>r$ and put with $D<r$, and it appears to be this discontinuity which leads to the logarithmic behavior. While this discontinuity is possible in the financial setting, it is unphysical, which perhaps explains why Tao [26-34] never encountered logarithmic behavior. 
To analyze the PDE (2.1), we will use an expansion in time which is an approach due to Tao [26-34], although in our case it is necessary to make a change of variables to transform (2.1) into a more standard diffusion equation together with a forcing term so that we can use Tao's method. We will proceed along the same lines as $[1,2,11,21]$ and make the change of variables $S=E e^{x}, t=T-2 \tau / \sigma^{2}$, and $V(S, t)=E v(x, \tau)$, which leads us to the diffusion-like PDE

$$
\frac{\partial v}{\partial \tau}=\frac{\partial^{2} v}{\partial x^{2}}+k_{2} \frac{\partial v}{\partial x}-k_{1} v-k_{3}
$$

where $k_{1}=2 r / \sigma^{2}$ and $k_{2}=2(r-D) / \sigma^{2}-1$ and $k_{3}=2 L /\left(E \sigma^{2}\right)$. Equation (2.3) is valid provided $\tau>0$ and it is optimal to hold the option, and must be solved together with the payoff at expiry, $v(x, 0)=\max \left(e^{x}-1,0\right)$ for a call and $\max \left(1-e^{x}, 0\right)$ for a put, and the condition on the free boundary,

$$
v=\frac{\partial v}{\partial x}=0
$$

At expiry the free boundary starts at $S=E$ or equivalently $x=0$. In the analysis that follows, strictly speaking (2.3) is valid only for those parameter values where it is advantageous to hold the option, so that at expiry we can only impose the initial condition on $x>0$ for the call and on $x<0$ for the put, and the initial condition becomes $v \rightarrow e^{x}-1$ as $\tau \rightarrow 0$ for the call and $v \rightarrow 1-e^{x}$ as $\tau \rightarrow 0$ for the put.

To tackle (2.3) and associated boundary and initial conditions, we will follow Tao and seek a series solution. While Tao expanded in powers of $\tau^{1 / 2}$, in the current problem, the discontinuity in the delta mentioned above means that we must include logs as well as powers of $\tau^{1 / 2}$ in the expansion, and this seems to be the rule when there is a discontinuity in the delta at the free boundary $[3,21]$. The series for $v(x, \tau)$ is therefore

$$
v(x, \tau)=\tau^{1 / 2} V_{1}^{(0)}(\xi)+\sum_{n=2}^{\infty} \sum_{m=0}^{\infty} \tau^{n / 2}(-\ln \tau)^{-m} V_{n}^{(m)}(\xi),
$$

which is the same form as for the American put with $D<r$ considered in [21], with $\xi=$ $x \tau^{-1 / 2} / 2$ a similarity variable. We assume that the free boundary is located at $x=x_{f}(\tau)$ which we also write as a series,

$$
x_{f}(\tau) \sim \sum_{n=1}^{\infty} \sum_{m=0}^{\infty} x_{n}^{(m)} \tau^{n / 2}(-\ln \tau)^{1-n / 2-m}
$$

with $x_{1}^{(0)}=\sqrt{2}$ for the installment call and $-\sqrt{2}$ for the put. The leading order scaling of $x_{f}(\tau) \sim x_{1}^{(0)}(-\tau \ln \tau)^{1 / 2}$, which is the same as for the vanilla put, is chosen because we need $\left|x_{f}(\tau)\right| \gg \sigma\left(\tau^{1 / 2}\right)$, and more specifically $\exp \left[-x_{f}^{2} / 4 \tau\right] \sim \mathcal{O}\left(\tau^{1 / 2}\right)$. With this expansion, it 
follows that on the free boundary we have

$$
\begin{gathered}
e^{-\xi^{2}}=\exp \left[-\frac{x_{f}^{2}}{4 \tau}\right] \sim \tau^{1 / 2} e^{x_{1}^{(2)} / \sqrt{2}}\left[1+\mathcal{O}\left(\ln ^{-1} \tau\right)\right] \\
\operatorname{erfc}(-\xi)=\operatorname{erfc}\left[-\frac{x_{f}}{2 \sqrt{\tau}}\right] \sim\left(\frac{2 \tau}{-\pi \ln \tau}\right)^{1 / 2} e^{x_{1}^{(2)} / \sqrt{2}}\left[1+\mathcal{O}\left(\ln ^{-1} \tau\right)\right]
\end{gathered}
$$

where erfc is the complementary error function and we have used the result that as $\zeta \rightarrow \infty$, $\operatorname{erfc}(\zeta) \sim\left(e^{-\zeta^{2}} / \zeta \sqrt{\pi}\right)\left[1+\sum_{m=1}^{\infty}\left((2 m-1) ! ! /\left(-2 \zeta^{2}\right)^{m}\right)\right]$

In our analysis, we substitute the assumed form for $v(x, \tau)(2.5)$ in the PDE (2.3) and group powers of $\tau^{1 / 2}$ and $\ln (-\tau)$. We find the following equations for the leading order terms at each power of $\tau^{1 / 2}$ in this expansion:

$$
\left[\frac{1}{4} \frac{d^{2}}{d \xi^{2}}+\frac{\xi}{2} \frac{d}{d \xi}-\frac{n}{2}\right] V_{n}^{(0)}= \begin{cases}0, & n=1 \\ -\frac{k_{2}}{2} V_{1}^{(0)^{\prime}}+k_{3}, & n=2 \\ -\frac{k_{2}}{2} V_{n-1}^{(0)^{\prime}}+k_{1} V_{n-2}^{(0)}, & n>2\end{cases}
$$

For the installment call, it is straightforward to write the solutions to (2.8) which satisfy the initial condition that $v(x, 0)=\max \left(e^{x}-1,0\right)$ for $x \geq 0$,

$$
\begin{gathered}
V_{1}^{(0)}=2 \xi+C_{1}^{(0)}\left(\frac{e^{-\xi^{2}}}{\sqrt{\pi}}+\xi[\operatorname{erfc}(-\xi)-2]\right) \\
V_{2}^{(0)}=2 \xi^{2}+k_{2}+1-k_{3}+C_{2}^{(0)}\left(\frac{2 \xi e^{-\xi^{2}}}{\sqrt{\pi}}+\left[2 \xi^{2}+1\right][\operatorname{erfc}(-\xi)-2]\right) \\
-C_{1}^{(0)} k_{2}\left(\frac{\xi e^{-\xi^{2}}}{\sqrt{\pi}}+\xi^{2}[\operatorname{erfc}(-\xi)-2]\right) .
\end{gathered}
$$

We would mention that since we can only impose the initial condition on $x>0$ for the call and on $x<0$ for the put, the limit $\tau \rightarrow 0$ means that $\xi \rightarrow+\infty$ for the call and $-\infty$ for the put. To impose the initial condition that $v \rightarrow e^{x}-1$ as $\tau \rightarrow 0$ for the call, we require that $\tau^{n / 2} V_{1}^{(n)} \rightarrow x^{n} / n !$, and we first set $e^{-\xi^{2}}=0$ and $\operatorname{erfc}(-\xi)=2$, then replace $\xi$ by $x \tau^{-1 / 2} / 2$, and finally take the limit $\tau \rightarrow 0$.

Next, we impose the conditions (2.4) at the free boundary on (2.9). To do this, we replace $x$ by (2.6), the series for $x_{f}(\tau)$, using the expressions (2.7) for $e^{-\xi^{2}}$ and $\operatorname{erfc}(-\xi)$ at the free boundary. This tells us that $C_{1}^{(0)}=1$ and $C_{2}^{(0)}=\left(k_{2}+1\right) / 2$, so that $(2.9)$ becomes

$$
\begin{aligned}
V_{1}^{(0)} & =\frac{e^{-\xi^{2}}}{\sqrt{\pi}}+\xi \operatorname{erfc}(-\xi), \\
V_{2}^{(0)} & =\frac{\xi e^{-\xi^{2}}}{\sqrt{\pi}}+\left(\xi^{2}+\frac{k_{2}+1}{2}\right) \operatorname{erfc}(-\xi)-k_{3} .
\end{aligned}
$$


Now that we have the leading order terms at each power of $\tau^{1 / 2}(2.10)$, we can comment further on the discontinuity in $(\partial v / \partial x)$. From $(2.10)$, at leading order, $(\partial v / \partial x) \sim$ $(1 / 2) \operatorname{erfc}\left(-x / 2 \tau^{1 / 2}\right)+O\left(\tau^{1 / 2}\right)$, which enables us to see the discontinuity: when $\tau=0$, $\operatorname{erfc}\left(-x / 2 \tau^{1 / 2}\right)=2$ for $x>0$, while on the free boundary $x_{f}(\tau)$, $\operatorname{erfc}\left(-x / 2 \tau^{1 / 2}\right) \sim$ $-\tau^{1 / 2}(-2 \pi \ln \tau)^{-1 / 2}$. The complementary error function provides immediate smoothing of this discontinuity, as $\tau$ increases from zero.

For the next terms in the expansion, at $\tau^{n / 2} /(-\ln \tau)$, we have the following equations:

$$
\left[\frac{1}{4} \frac{d^{2}}{d \xi^{2}}+\frac{\xi}{2} \frac{d}{d \xi}-\frac{n}{2}\right]= \begin{cases}0, & n=2, \\ -\frac{k_{2}}{2} V_{2}^{(1)^{\prime}}(\xi), & n=3, \\ -\frac{k_{2}}{2} V_{n-1}^{(1)^{\prime}}(\xi)+k_{1} V_{n-2}^{(1)}, & n>3 .\end{cases}
$$

It should be noted that (2.11) do not involve the leading order terms $V_{n}^{(0)}$. The solution at the first order is

$$
V_{2}^{(1)}=C_{2}^{(1)}\left(\frac{2 \xi e^{-\xi^{2}}}{\sqrt{\pi}}+\left[2 \xi^{2}+1\right][\operatorname{erfc}(-\xi)-2]\right)
$$

With our expression for the free boundary (2.6), the conditions (2.4) on the free boundary applied to (2.12) become at leading order

$$
\begin{gathered}
-k_{3}-2 C_{2}^{(1)}+O\left[(-\ln \tau)^{-1}\right]=0, \\
2^{5 / 2} C_{2}^{(1)}+\sqrt{\frac{2}{\pi}} e^{x_{1}^{(1)} / \sqrt{2}}+O\left[(-\ln \tau)^{-1}\right]=0,
\end{gathered}
$$

so that $C_{2}^{(1)}=-k_{3} / 2$ and $x_{1}^{(1)}=\sqrt{2} \ln \left(2 \sqrt{\pi} k_{3}\right)$. We now know the behavior of the free boundary in the limit $\tau \rightarrow 0$,

$$
\begin{aligned}
x_{f}(\tau) & \sim \sqrt{-2 \tau \ln \tau}\left(1-\frac{\ln \left(4 \sqrt{\pi} L /\left(E \sigma^{2}\right)\right)}{\ln \tau}+O\left(\ln ^{-2} \tau\right)\right)+O(\tau), \\
S_{f}(t) \sim E \exp [ & \sigma \sqrt{-(T-t) \ln \left[\frac{\sigma^{2}(T-t)}{2}\right]} \\
& \left.\times\left(1-\frac{\ln \left(4 \sqrt{\pi} L /\left(E \sigma^{2}\right)\right)}{\ln \left[\sigma^{2}(T-t) / 2\right]}+O\left(\ln ^{-2}\left[\frac{\sigma^{2}(T-t)}{2}\right]\right)\right)+O(T-t)\right] .
\end{aligned}
$$

Our analysis for the call is now complete, so we now turn our attention to the installment put, for which the analysis is very similar, since of course the put and the call obey the same PDE but with different boundary conditions, and we find that the behavior of 
the free boundary for the installment put in the limit $\tau \rightarrow 0$ is

$$
\begin{aligned}
x_{f}(\tau) \sim & -\sqrt{-2 \tau \ln \tau}\left(1-\frac{\ln \left(4 \sqrt{\pi} L /\left(E \sigma^{2}\right)\right)}{\ln \tau}+O\left(\ln ^{-2} \tau\right)\right)+O(\tau), \\
S_{f}(t) \sim E \exp [ & -\sigma \sqrt{-(T-t) \ln \left[\frac{\sigma^{2}(T-t)}{2}\right]} \\
& \left.\times\left(1-\frac{\ln \left(4 \sqrt{\pi} L /\left(E \sigma^{2}\right)\right)}{\ln \left[\sigma^{2}(T-t) / 2\right]}+O\left(\ln ^{-2}\left[\frac{\sigma^{2}(T-t)}{2}\right]\right)\right)+O(T-t)\right] .
\end{aligned}
$$

We note that the behavior of installment puts and calls close to expiry is very similar, with the leading order behavior in the limit $\tau \rightarrow 0$ of $x_{f}(\tau)$ for the put being minus that for the call, so that there is an asymptotic put-call parity of sorts. This suggests that there is some sort of put-call symmetry for installment options, perhaps along the lines of that for vanilla Americans $[8,22]$, and it would be interesting to find the exact forms of this symmetry for these and other American-style exotics.

The problem we have considered above has a couple of interesting limits. For $0<L<$ $\infty$, the free boundary will always start at $S=E$ at $\tau=0$. If $L=0$, so that no installments are paid, obviously the option should never be allowed to lapse, as the expected net present value of the contract would always be positive, so when $L=0$, the free boundary will be $S_{f}(\tau) \equiv 0$ for the call and $S_{f}(\tau) \equiv \infty$ for the put. Similarly, if $L=\infty$, the option should always be abandoned, as the expected net present value of the contract would always be negative, so when $L=\infty$, the free boundary will be $S_{f}(\tau) \equiv \infty$ for the call and $S_{f}(\tau) \equiv 0$ for the put. As we take the limit $L \rightarrow 0+$, the free boundary will always start from $S=E$ at $\tau=0$, and therefore we will not recover the case $L=0$ as we take the limit, and this is what is known as a singular perturbation problem. As a result of this, as pointed out by a referee, our expressions for the location of the free boundary (2.14), (2.15) are singular in the limit $L \rightarrow 0+$. The same is true of the limit $L \rightarrow \infty$. It follows from this that, strictly speaking, the analysis presented here is valid when $L \sim O(1)$.

There are several methods to tackle singular perturbation problems such as this, and these methods are invariably long and complicated, and beyond the scope of the present study, with the most popular being the method of multiple scales. With an installment option, the premium is paid continuously at a rate $L$, so in time $d t$ an amount $L d t$ is paid. To consider the limit $L \rightarrow 0+$, it would be necessary to add a slow time scale $\hat{\tau}$ such that $L d \hat{\tau} \sim \mathcal{O}(1)$. If we write $L=\varepsilon \hat{L}$, with $\hat{L} \sim \mathcal{O}(1)$ and $0<\varepsilon \ll 1$, then the slow time scale would be $\hat{\tau}=\varepsilon^{-1} \tau$, and (2.3) would become

$$
\frac{\partial v}{\partial \tau}+\varepsilon \frac{\partial v}{\partial \hat{\tau}}=\frac{\partial^{2} v}{\partial x^{2}}+k_{2} \frac{\partial v}{\partial x}-k_{1} v-\varepsilon \hat{k}_{3}
$$

where $\hat{k}_{3}=\varepsilon^{-1} k_{3} \sim \mathcal{O}(1)$. We would then expand $v(x, \tau, \hat{\tau})$ as a series in $\varepsilon$, with the $\varepsilon^{0}$ term simply the value of a European option. The free boundary $x_{f}(\hat{\tau})$ in this case would depend on only the slow time scale $\hat{\tau}$. 


\section{Discussion}

In the previous section, we used an asymptotic expansion of the governing PDE to study the behavior of installment options close to expiry, and in particular found expressions (2.14), (2.15) for the location of the free boundary, along with expressions (2.6), (2.10), (2.12) for the value of the call option in that limit. These two sets of expressions constitute the principal result of this paper. It is interesting to note that, provided $L$, which represents the continual input of cash in the form of installment payments, is positive, the location of the free boundary close to expiry is of the form $x_{f}(\tau) \sim \sqrt{\tau(-\ln \tau)}$, which is the same behavior as we found using an integral equation approach in [4], and also the same as that for the American put with $D<r$ and the American call with $D>r[2,5,13,16,17,21,25]$. This differs from the $x_{f}(\tau) \sim x_{1} \sqrt{\tau}$ behavior for the American put with $D>r$ and the American call with $D<r$ which was also the behavior encountered most often by Tao [26-34], who pioneered the method used here, in his studies of Stefan problems arising in melting and solidification. Although Tao encountered several behaviors other than the $\sqrt{\tau}$ behavior, he did not come across the $\sqrt{\tau(-\ln \tau)}$ behavior found both here and with American options for the parameter ranges discussed above. We suspect that this logarithmic behavior is caused by the discontinuity in $(\partial V / \partial S)$ which we discussed earlier, and since this discontinuity is unphysical, Tao did not encounter it.

\section{References}

[1] G. Alobaidi and R. Mallier, Asymptotic analysis of American call options, International Journal of Mathematics and Mathematical Sciences 27 (2001), no. 3, 177-188.

[2] _ On the optimal exercise boundary for an American put option, Journal of Applied Mathematics 1 (2001), no. 1, 39-45.

[3] _ American lock-in options close to expiry, International Journal of Applied Mathematics 13 (2003), no. 4, 409-429.

[4] G. Alobaidi, R. Mallier, and A. S. Deakin, Laplace transforms and installment options, Mathematical Models \& Methods in Applied Sciences 14 (2004), no. 8, 1167-1189.

[5] G. Barles, J. Burdeau, M. Romano, and N. Samsoen, Critical stock price near expiration, Mathematical Finance 5 (1995), no. 2, 77-95.

[6] F. Black and M. Scholes, The pricing of options and corporate liabilities, The Journal of Political Economy 81 (1973), no. 3, 637-654.

[7] D. M. Chance, Essays in Derivatives, John Wiley \& Sons, New York, 1998.

[8] M. Chesney and R. Gibson, State space symmetry and two factor option pricing models, Advances in Futures and Operations Research 8 (1993), 85-112.

[9] M. H. A. Davis, W. Schachermayer, and R. G. Tompkins, Pricing, no-arbitrage bounds and robust hedging of instalment options, Quantitative Finance 1 (2001), no. 6, 597-610.

[10] _ Installment options and static hedging, The Journal of Risk Finance 3 (2002), no. 2, $46-52$.

[11] J. N. Dewynne, S. D. Howison, I. Rupf, and P. Wilmott, Some mathematical results in the pricing of American options, European Journal of Applied Mathematics 4 (1993), no. 4, 381-398.

[12] A. R. Dixit and R. S. Pindyck, Investment Under Uncertainty, Princeton University Press, New Jersey, 1994.

[13] J. D. Evans, R. Kuske, and J. B. Keller, American options of assets with dividends near expiry, Mathematical Finance 12 (2002), no. 3, 219-237.

[14] J. Hakala and U. Wystup, Foreign Exchange Risk: Models, Instruments and Strategies, Risk, London, 2002. 
[15] F. Karsenty and J. Sikorav, Installment plan, Risk Magazine 6 (1993), 36-40.

[16] C. Knessl, A note on a moving boundary problem arising in the American put option, Studies in Applied Mathematics 107 (2001), no. 2, 157-183.

[17] R. E. Kuske and J. B. Keller, Optimal exercise boundary for an American put option, Applied Mathematical Finance 5 (1998), no. 2, 107-116.

[18] Y. K. Kwok, Mathematical Models of Financial Derivatives, Springer Finance, Springer, Singapore, 1998.

[19] S. Majd and R. S. Pindyck, Time to build, option value, and investment decisions, Journal of Financial Economics 18 (1987), no. 1, 7-27.

[20] R. Mallier and G. Alobaidi, Laplace transforms and American options, Applied Mathematical Finance 7 (2000), no. 4, 241-256.

[21] - The American put option close to expiry, Acta Mathematica Universitatis Comenianae. New Series 73 (2004), no. 2, 161-174.

[22] R. McDonald and M. Schroder, A parity result for American options, The Journal of Computational Finance 1 (1998), no. 3, 5-13.

[23] R. C. Merton, Theory of rational option pricing, Bell Journal of Economics and Management Science 4 (1973), no. 1, 141-183.

[24] P. A. Samuelson, Rational theory of warrant pricing, Industrial Management Review 6 (1965), no. $2,13-31$.

[25] R. Stamicar, D. Ševčovič, and J. Chadam, The early exercise boundary for the American put near expiry: numerical approximation, The Canadian Applied Mathematics Quarterly 7 (1999), no. 4, 427-444.

[26] L. N. Tao, The Stefan problem with arbitrary initial and boundary conditions, Quarterly of Applied Mathematics 36 (1978), no. 3, 223-233.

[27] _ Free boundary problems with radiation boundary conditions, Quarterly of Applied Mathematics 37 (1979), no. 1, 1-10.

[28] _ On free boundary problems with arbitrary initial and flux conditions, Zeitschrift für Angewandte Mathematik und Physik 30 (1979), no. 3, 416-426.

[29] _ On solidification problems including the density jump at the moving boundary, The Quarterly Journal of Mechanics and Applied Mathematics 32 (1979), no. 2, 175-185.

[30] - On solidification of a binary alloy, The Quarterly Journal of Mechanics and Applied Mathematics 33 (1980), no. 2, 211-225.

[31] The analyticity of solutions of the Stefan problem, Archive for Rational Mechanics and Analysis 72 (1980), no. 3, 285-301.

[32] - The exact solutions of some Stefan problems with prescribed heat flux, Journal of Applied Mechanics. Transactions of the ASME 48 (1981), no. 4, 732-736.

[33] _ The Cauchy-Stefan problem, Acta Mechanica 45 (1982), no. 1-2, 49-64.

[34] , The Stefan problem with an imperfect thermal contact at the interface, Journal of Applied Mechanics 49 (1982), no. 4, 715-720.

[35] P. Wilmott, Paul Wilmott on Quantitative Finance, John Wiley \& Sons, Chichester, 2000.

G. Alobaidi: Department of Mathematics and Statistics, American University of Sharjah,

P.O. Box 26666, Sharjah, United Arab Emirates

E-mail address: galobaidi@aus.edu

R. Mallier: Department of Applied Mathematics, University of Western Ontario, London, ON, Canada N6A 5B7

E-mail address: rolandmallier@hotmail.com 


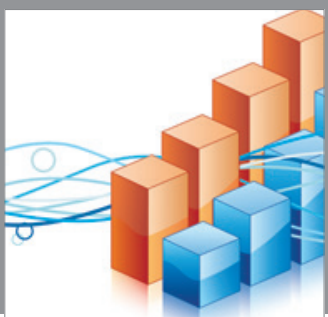

Advances in

Operations Research

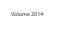

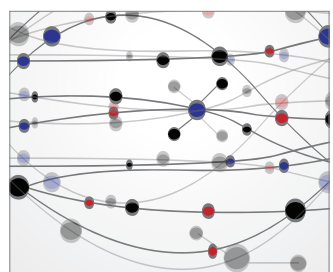

\section{The Scientific} World Journal
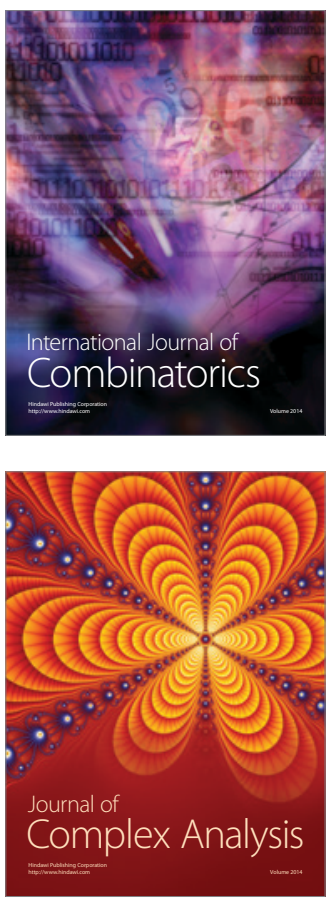

International Journal of

Mathematics and

Mathematical

Sciences
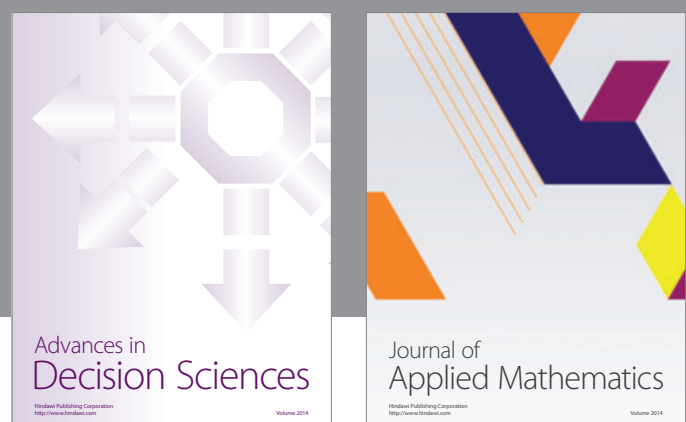

Journal of

Applied Mathematics
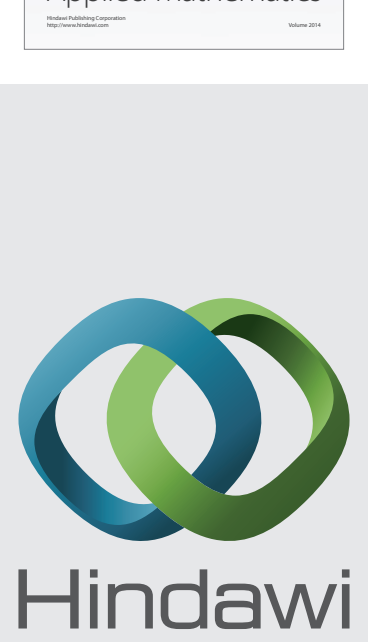

Submit your manuscripts at http://www.hindawi.com
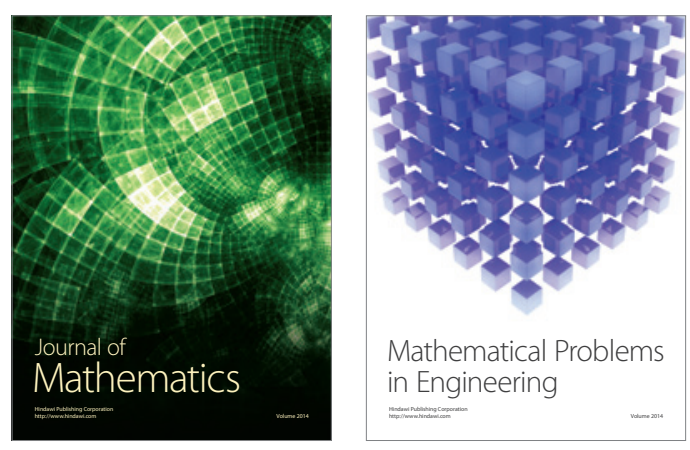

Mathematical Problems in Engineering
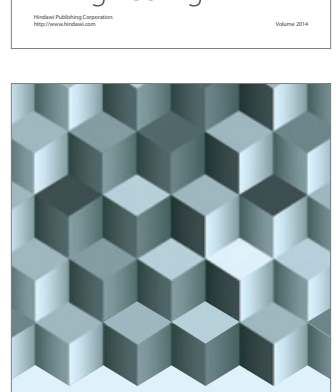

Journal of

Function Spaces
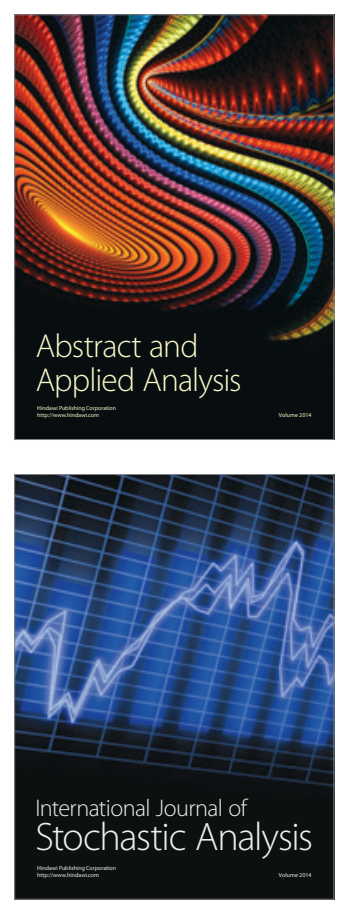

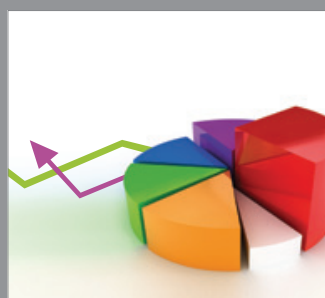

ournal of

Probability and Statistics

Promensencen
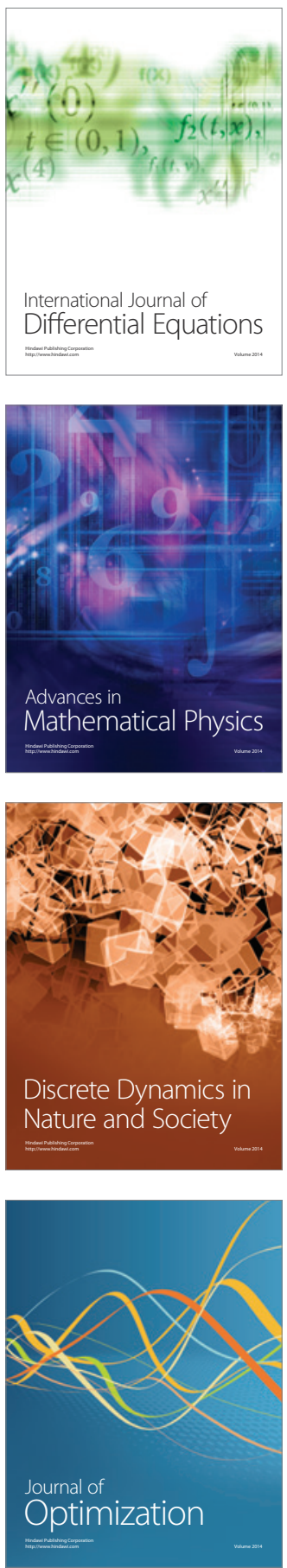